\title{
Reliability oriented thermal management of aircraft power converters
}

\author{
Jayakrishnan Harikumaran ${ }^{1}$, Giampaolo Buticchi ${ }^{1,2}$, Giovanni MIgliazza ${ }^{3}$, Patrick Wheeler ${ }^{1}$, and Michael Galea ${ }^{1,2}$ \\ ${ }^{1}$ Department of Power Electronics Machines and Control, University of Nottingham \\ ${ }^{2}$ Zhejiang Key Laboratory on the More Electric Aircraft Technology, University of Nottingham Ningbo China \\ ${ }^{3}$ Department of Sciences and Methods for Engineering, University of Modena and Reggio Emilia
}

\begin{abstract}
On board electrical power generation continue to increase as more aircraft systems are being electrified. Increasingly power converters are the interface of power generation and electrical loads in aircrafts. Reliability of aircraft power electronics is essential for high reliability and mission critical applications. Along with wear-out failure mechanisms, random failure modes must also be addressed to realize high reliability aerospace power converters. A dominant random failure mode in aerospace environment is due to cosmic rays (CR). In this work, a thermal management approach is proposed to maximize reliability of the converters considering $C R$ failure mode. The proposed thermal controller is validated with an experimental setup. In the discussion, the trade-off involved between wear-out and CR induced failure mode is addressed.

Index Terms-Cosmic ray failure, Reliability, Thermal control, Power electronics
\end{abstract}

\section{INTRODUCTION}

Aircraft designers are increasingly incorporating electrical sub-systems in order to meet pollution reduction targets set by governmental and regulatory bodies [1]. Substituting hydraulic systems, pneumatic air system etc. with electrical counter parts simplify aircraft architecture while achieving overall better aircraft fuel efficiency [2]. In the latest generation aircrafts, on board power generation exceeds $1 \mathrm{MW}$ [3].

As described in [4], the on-board electrical architecture of more and all electric aircraft includes electrical generation and electric propulsion. The eFanX demonstration project from Airbus-Rolls Royce consortium proposes using this architecture. Critical role of drive converters for future electric aircrafts is highlighted as they are part of mission critical propulsion system. Hence it is vital to achieve high reliability of aviation drive converters.

Prior works have focused on wear-out reliability estimation of power converters - railway traction converters [5], electric vehicle traction [6]. Reliability of converter system would be over-estimated if random failures are not considered [7]. Random failures cannot be ignored for power converters operating in high reliability applications. Under aerospace operating conditions, CR induced failures is a dominating failure mode in power converters.

This work was supported by the INNOVATIVE Doctoral Program. The INNOVATIVE program is supported in part by the Marie Curie Initial Training Networks action under Grant 665468, in part by the Institute for Aerospace Technology, University of Nottingham, and in part by the University of Nottingham Propulsion Futures Beacon.
Thermal control approaches have been proposed in literature to minimize thermal ageing of power components [8], [9]. A typical design aim of thermal control for reliability management is to minimize junction temperature cycles of semiconductors or equalize junction temperature of semiconductors and/or capacitors to maximize system lifetime.

Due to semiconductor properties $\mathrm{CR}$ induced failure rate accelerate, when junction temperature decreases. Hence there is a requirement to control junction temperature of semiconductors in an optimal range to balance failure rates due to wear-out failure and CR failures. The main contribution of this work is the proposed active thermal controller. Converter thermal management is performed taking into account estimated CR flux at altitude. An experimental setup validates the proposed approach. In discussion section, impact of thermal control on CR failure rate is quantified and compared.

\section{Single Event Burnout (SEB) of SEMICONDUCTORS DUE TO COSMIC RAYS}

Impact of atmospheric radiations were initially observed in semiconductor memory chips. Random bit flips in Dynamic Random Access Memory (DRAM) chips were eventually related to background atmospheric nucleon flux. The classic work from IBM on terrestrial cosmic rays [10] gives a comprehensive review on SEE (single event error) mechanisms due to nucleon flux in semiconductor chips. SEE is not a breakdown event as there is not enough energy at low voltages.

In case of high voltage semiconductors (above 300V), a high electrical field is present in the bulk of semiconductors, during reverse blocking mode. SEB in power semiconductors are mainly caused by high energy neutrons. The capture of neutrons by atoms in the semiconductors ( $\mathrm{Si}$ in case of IGBTs, $\mathrm{Si}$ or $\mathrm{C}$ in case of $\mathrm{SiC}$ devices) causes nuclear reactions. The resultant nuclear fragments deposit a charge in semiconductor bulk and acts as seed for CR induced effects. The deposited charge may develop into a streamer of electrons and holes due to electrical field. This leads to localized electric field spikes in semiconductor. A runaway process ensues causing avalanche multiplication of charge carriers. Eventually a short circuit develops leading to sudden device destruction [11], [12]. 


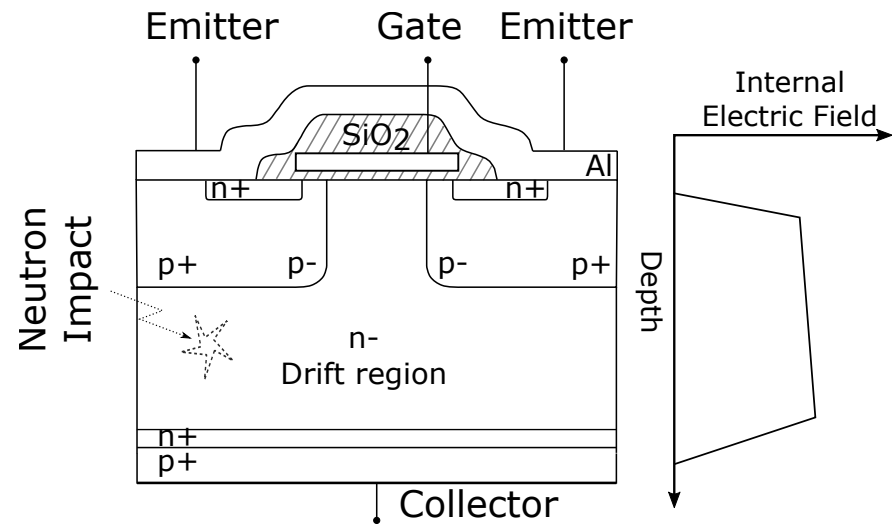

Fig. 1. IGBT Cross section and internal electric field in blocking mode

\section{A. Atmospheric nucleon flux at aircraft altitudes}

Interaction of "Cosmic rays" with earth's atmosphere (mainly nitrogen and oxygen molecules) generates secondary ionizing particles [13]. The most damaging of secondary particles for avionic systems is found to be neutrons [14]. Hess in his pioneering work in 1959 quantified neutron flux profile in atmosphere [15]. Boeing published neutron and other particle flux density and energy spectra variations due to altitude and latitude in [16]. From these prior works, neutron flux is found to increase with altitude and reach its maximum around 60000 feet (named Pfotzer maximum). CR intensity is also dependent on latitude - with flux intensity almost double near poles compared to intensity at equator.

It has been reported in literature that the CR intensity at aircraft altitudes are up to 300 times of that at sea level. The atmospheric neutron flux profile is summarized in [10] based on numerous studies. This work along with the IEC standard 62396 provides guidelines for avionics system designers [17]. The neutron flux at any point in the atmosphere can be quantified if the following quantities are known - the latitude and longitude of the location, atmospheric rigidity of that location and altitude of interest. IEC62396 recommends a scaling procedure to estimate neutron flux density at any point in atmosphere by scaling the reference value at a known location and altitude. A neutron flux of $6000 \frac{n}{\mathrm{~cm}^{2} h}$ is reported for neutrons with energy $>10 \mathrm{MeV}$ at an altitude of 40000 ft. at $45^{\circ}$ latitude [18].

\section{B. SEB failure rate estimation}

As CR failure is a complex phenomenon, analytical models are not readily available. Neutron flux and reverse blocking voltage and device physical properties determine CR failure rate. The relation to neutron flux is linear while relation to blocking voltage and device characteristics is highly nonlinear.

An analytical expression was proposed by ABB to model rate of IGBT failure depending on blocking voltage, temperature, altitude, (1) [19]. The temperature dependency arises as semiconductor blocking voltage reduces with temperature. Electron charge cloud density in semiconductor bulk increases when temperature is reduced. Consequentially, avalanche breakdown is induced at lower voltages as there is higher chance of impact ionization. As reported by $A B B$, the temperature and altitude dependency is module independent for Si devices while voltage dependency is experimentally curve fitted for particular modules.

IEC-62396-4 specifically deals with SEB failure rate characterization of high voltage power devices in aircraft applications. According to IEC, experimental measurements of CR failures reported in FIT (failure in time) per unit area at different reversing blocking voltage levels at a reference neutron flux level can be scaled with actual neutron flux to obtain FIT figures at aerospace operating conditions. 1 FIT is equivalent to 1 failure every $10^{9}$ hours of operation.

This method would be adopted in this work to obtain the FIT rate without accounting for temperature effects. CR failure rates for selected modules are reported in [20], [21].

\section{CASE STUDY - COSMIC RAY FAILURE RATE ESTIMATION}

In this section, case study results of CR SEB rate estimation for a particular aerospace starter generator drive converter [22], is presented. Drive converter in above work is a 3LNPC converter. Atmospheric model is described followed by estimated cosmic ray failure rate for the converter system. Only CR failure rate is discussed here. A detailed reliability study including wear-out failures is being carried out for future publication.

\section{A. Atmospheric model}

As mentioned before, neutron flux intensity is key parameter necessary for CR failure rate calculation once system voltage is known. Altitude profile is an indirect measure of neutron flux at actual operating conditions. Temperature impact on SEB rate necessitates estimation of temperature profile. In a prior work from same authors [23], atmospheric profile a typical short haul flight was modeled based on US standard atmosphere 1976 [24]. An altitude/temperature profile from above referred work is reproduced in Fig. 2. The flight profile modelled includes 1 hour of cruising at 30000 feet. Necessary mission profile data - altitude and temperature profile and operating time are thus generated.

\section{B. $C R$ failure rate for $S G$ converter system}

Experimental results in [21] highlight, Si IGBTs suffer from cosmic ray failures above $60 \%$ of blocking voltage rating which follow recommendations on CR failures from Semikron [12]. A DC voltage of $810 \mathrm{~V}$ is considered here and hence 650V IGBTs (SEMiX155MLI07E4) are selected for the drive converter. Neutron flux intensity at $30000 \mathrm{ft}$. is 130 times higher than at reference conditions [18]. CR failure rate of 9.9 FIT is tabulated based on experimental FIT rates from [21] scaled to account for increased neutron flux intensity. Above FIT rate is valid for a semiconductor in blocking mode during entire operation. Due to modulation, scaling factors 


$$
\lambda\left(V_{D C}, T_{j}, h\right)=C_{3} \cdot \exp \left(\frac{C_{2}}{C_{1}-V_{D C}}\right) \cdot \exp \left(\frac{25-T_{j}}{47.6}\right) \cdot \exp \left(\frac{1-\left(1-\frac{h}{44300}\right)^{5} \cdot 26}{0.143}\right)
$$

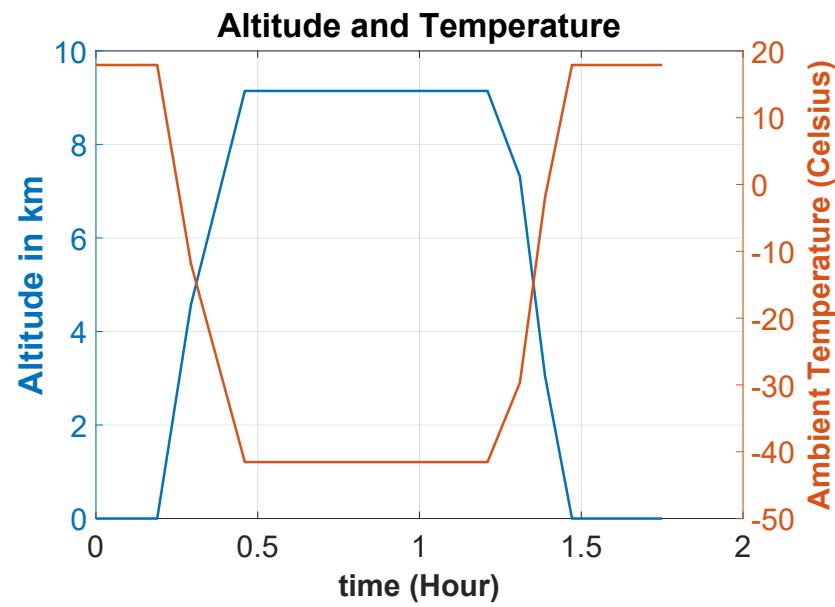

Fig. 2. Altitude and temperature variation during flight

corresponding to percentage of time spent in blocking mode are applied for the switches:

- $75 \%$ for the outer switches in 3L-NPC;

- $25 \%$ for the inner switches in 3L-NPC.

Cumulative CR failure rate of 3L-NPC converter is $0.0013 \%$ /year.

1) Motivation for thermal controller: The CR failure rate from section III-B is derived at a reference junction temperature of $25{ }^{\circ} \mathrm{C}$. The temperature dependence from (1) shows that the cosmic ray failure rate at a junction temperature of 70 ${ }^{\circ} \mathrm{C}$ reduces by $39 \%$, while the failure rate increases by $209 \%$ when junction temperature is $-10{ }^{\circ} \mathrm{C}$. Considering ambient temperature reduces with altitude, temperature dependence must also be accounted for in reliability estimations.

\section{THERMAL CONTROLLER FOR AEROSPACE CONDITIONS}

A thermal controller is proposed with the aim of maintaining junction temperature of semiconductors in an optimum range. There are many handles to vary junction temperature in a switching power converter - switching frequency, cooling system design, device selection etc. Switching frequency variation is utilized in this work. It must be noted that other methods are also feasible.

\section{A. Thermal controller structure}

The thermal controller is implemented as a simple Pcontroller with saturation for the maximum and minimum switching frequency values. The temperature set point could be generated in multiple ways - based on measured atmospheric flux at altitude stored in lookup tables being one of the simplest methods. The thermal controller structure is shown in Fig. 3.

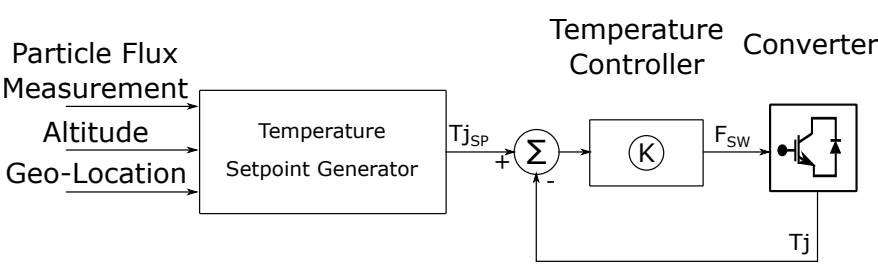

Fig. 3. Junction temperature controller

\section{B. Experimental setup}

Performance of thermal controller is experimentally validated using a $2 \mathrm{~L} \mathrm{SiC}$ drive converter. The test setup is shown in Fig. 4. The setup includes a DC power source to drive $\mathrm{SiC}$ inverter, an RL load with an inducatance of $0.5 \mathrm{mH}$ and a variable resistor bank, Hioki PW3390 power analyzer, oscilloscope and $\mathrm{SiC}$ inverter. $\mathrm{SiC}$ module used is CCS050M12CM2 from CREE rated at $1.2 \mathrm{kV}$ and $87 \mathrm{~A}$ and DC link is composed of 20 capacitors from TDK Epcos (B32652A4474J000) rated at $470 \mathrm{nF}, 400 \mathrm{~V}$. The fundamental frequency of AC output is $100 \mathrm{~Hz}$ and on chip NTC is used to measure case temperature of the module.

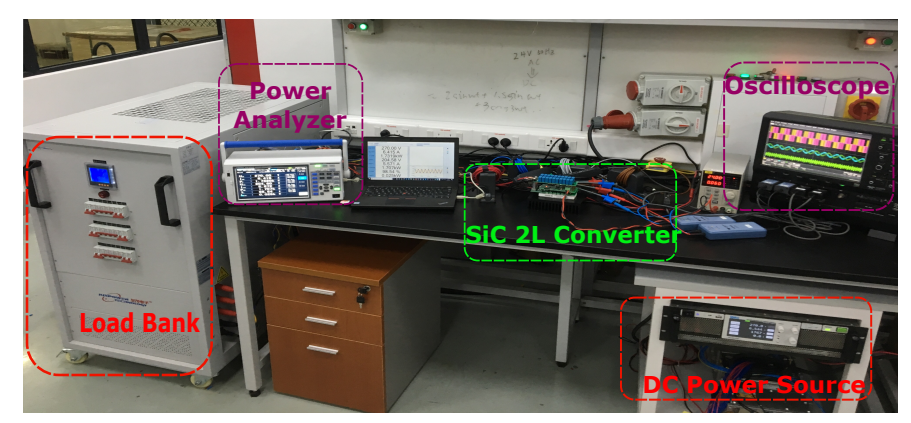

Fig. 4. Test Setup for thermal controller

The on-chip NTC is characterised to obtain resistance variation relationship with temperature. On-chip ADC samples temperature of the module to be used in thermal controller.

\section{Experimental results}

The controller test result is captured in Fig. 5 and Fig. 6. The module temperature is measured and the switching frequency is varied by the controller to maintain the module temperature in the control range. The switching frequency limits are set to $10 \mathrm{kHz}$ and $50 \mathrm{kHz}$.

The system unreliability for $3 \mathrm{~L}$ NPC converter is shown and a detailed reliability analysis would be published in a future work. It can be seen that change in failure rate have significant impact on the Bxx life as prescribed in aerospace safety requirements. 


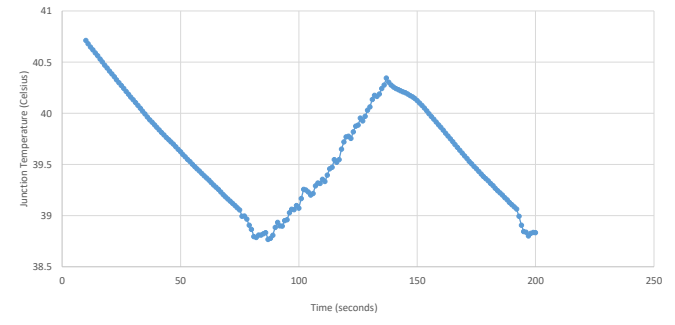

Fig. 5. SiC Module temperature

\section{Discussion}

The impact on failure rate due to active thermal control is visualized in Fig. 7. A temperature variation of $10{ }^{\circ} \mathrm{C}$ with thermal control is modelled.

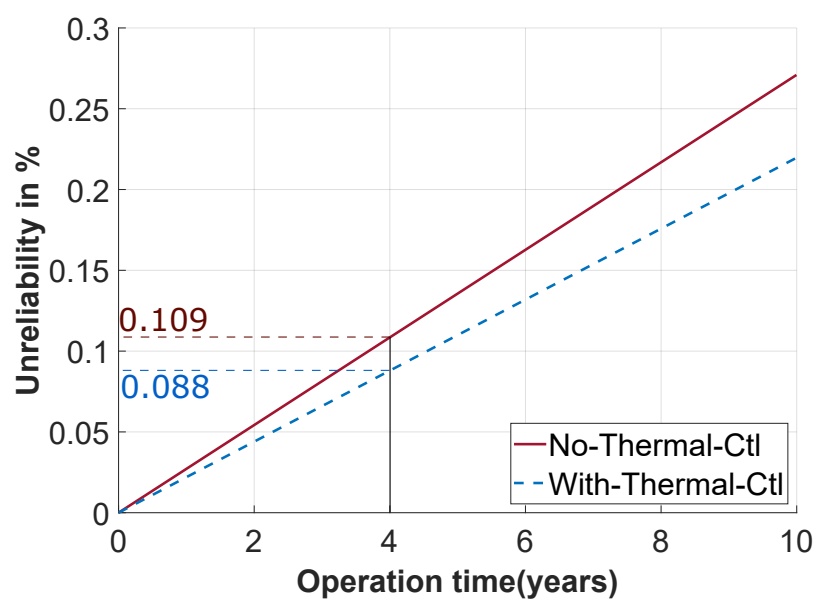

Fig. 7. Thermal control variation of CR Unreliability

The $B_{\text {life }}$ at 4 years shows a significant variation even with a 10 degree modification in junction temperature. It must be noted that, reliability requirements in aerospace applications requires very low $B_{\text {life }}$ (proportion of systems failing by a given time). Hence a variation of $25 \%(0.088 \%$ to $0.108 \%)$ in unreliability is significant. The significance of CR failure rate controller also arises from variation of neutron flux with latitude. At high latitudes, CR intensity is more than double of that at equatorial regions. This effect could also be mitigated to some extent by thermal control.

For comprehensiveness, Reliability Block Diagram (RBD) method to quantify converter reliability is shown in Fig. 8

Converter system reliability is a combination of reliability of various sub-components. In case of semiconductors, unreliability due to wear-out and $\mathrm{CR}$ must be included. A $10^{\circ} \mathrm{C}$ variation in junction temperature roughly equates to doubling of effective thermal wear-out damage [25]. Hence there is a trade-off involved as mentioned earlier with thermal control.

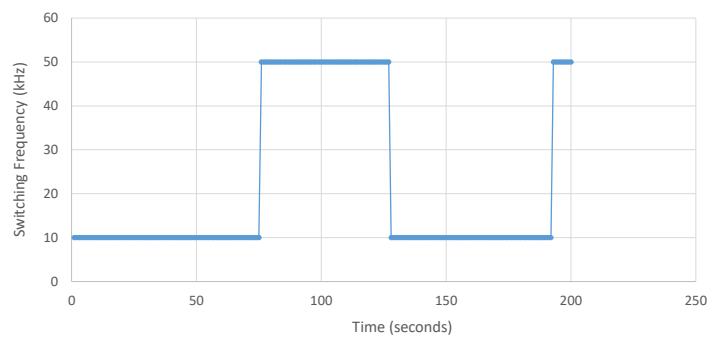

Fig. 6. Converter switching frequency

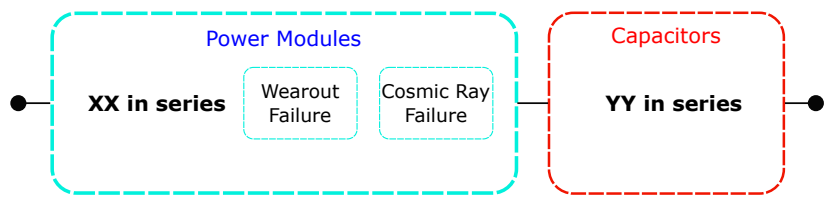

Fig. 8. Reliability block diagram

Under aerospace operating conditions, CR failures tend to dominate as the ambient temperatures are very low resulting in low wear-out damage. Such a combined optimization is necessary to maximize converter reliability in $\mathrm{CR}$ failure dominated systems. It can also be concluded that voltage derating for aerospace applications is particularly significant for semiconductors.

\section{CONCLUSION}

Cosmic ray failures is an important failure mode to consider for aerospace power converters. Temperature dependence of $\mathrm{CR}$ failure rate accentuates $\mathrm{CR}$ failures at high altitude conditions. A thermal controller is proposed to maintain junction temperature of semiconductors in an optimum range to maximize converter reliability.

\section{REFERENCES}

[1] IATA, DLR, and G. I. of Technology, IATA TECHNOLOGY ROADMAP 2013, International Air Transport Association, 2013.

[2] M. J. Provost, "The more electric aero-engine: a general overview from an engine manufacturer," in 2002 International Conference on Power Electronics, Machines and Drives (Conf. Publ. No. 487), June 2002, pp. 246-251.

[3] V. Madonna, P. Giangrande, and M. Galea, "Electrical power generation in aircraft: Review, challenges, and opportunities," IEEE Transactions on Transportation Electrification, vol. 4, no. 3, pp. 646-659, Sep. 2018.

[4] G. Buticchi, S. Bozhko, M. Liserre, P. Wheeler, and K. Al-Haddad, "On-board microgrids for the more electric aircraft- technology review," IEEE Transactions on Industrial Electronics, vol. 66, no. 7, pp. 55885599, July 2019.

[5] X. Perpiñà, J. Serviere, L. Navarro, M. Mermet-Guyennet, M. Vellvehi, and X. Jordà, Reliability and Lifetime Prediction for IGBT Modules in Railway Traction Chains. INTECH Open Access Publisher, 2012. [Online]. Available: https://books.google.co.uk/books?id=LkLYoAEACAAJ

[6] D. Hirschmann, D. Tissen, S. Schroder, and R. W. D. Doncker, "Reliability prediction for inverters in hybrid electrical vehicles," IEEE Transactions on Power Electronics, vol. 22, no. 6, pp. 2511-2517, Nov 2007. 
[7] U. Scheuermann and M. Junghaenel, "Limitation of power module lifetime derived from active power cycling tests," in CIPS 2018; 10th International Conference on Integrated Power Electronics Systems, March 2018, pp. 1-10.

[8] M. Andresen, J. Kuprat, V. Raveendran, J. Falck, and M. Liserre, "Active thermal control for delaying maintenance of power electronics converters," Chinese Journal of Electrical Engineering, vol. 4, no. 3, pp. 13-20, September 2018.

[9] M. Andresen, K. Ma, G. Buticchi, J. Falck, F. Blaabjerg, and M. Liserre, "Junction temperature control for more reliable power electronics," IEEE Transactions on Power Electronics, vol. 33, no. 1, pp. 765-776, Jan 2018.

[10] J. F. Ziegler, "Terrestrial cosmic rays," IBM Journal of Research and Development, vol. 40, no. 1, pp. 19-39, Jan 1996.

[11] U. Scheuermann and U. Schilling, "Impact of device technology on cosmic ray failures in power modules," IET Power Electronics, 052016.

[12] D. U. Schilling, Cosmic Ray Failures in Power Electronics, Semikron Gmbh, 2017.

[13] T. Sato, "Analytical model for estimating terrestrial cosmic ray fluxes nearly anytime and anywhere in the world: Extension of parma/expacs," PloS one, vol. 10, no. 12, p. e0144679, 2015. [Online]. Available: http://europepmc.org/articles/PMC4682948

[14] G. Soelkner, "Ensuring the reliability of power electronic devices with regard to terrestrial cosmic radiation," $\mathrm{Mi}$ croelectronics Reliability, vol. 58, pp. 39 - 50, 2016 , reliability Issues in Power Electronics. [Online]. Available: http://www.sciencedirect.com/science/article/pii/S0026271415302663

[15] H.Wade Patterson, W. N. Hess, B. J. Mayer, and R. W. Wallace, "The flux and spectrum of cosmic-ray-produced neutrons as a function of altitude," 1959.

[16] E. Normand and T. J. Baker, "Altitude and latitude variations in avionics seu and atmospheric neutron flux," IEEE Transactions on Nuclear Science, vol. 40, no. 6, pp. 1484-1490, Dec 1993.

[17] I. E. Commission, Process management for avionics - Atmospheric radiation effects, International Electrotechnical Commission, 2017.

[18] R. Edwards, C. Dyer, and E. Normand, "Technical standard for atmospheric radiation single event effects, (see) on avionics electronics," in 2004 IEEE Radiation Effects Data Workshop (IEEE Cat. No.04TH8774), July 2004, pp. 1-5.

[19] N. Kaminski, Failure Rates of HiPak Modules Due to Cosmic Rays Application Note 5SYA 2042-02, ABB Switzerland Ltd, 2004.

[20] G. Consentino, M. Laudani, G. Privitera, C. Pace, C. Giordano, J. Hernandez, and M. Mazzeo, "Effects on power transistors of terrestrial cosmic rays: Study, experimental results and analysis," in 2014 IEEE Applied Power Electronics Conference and Exposition - APEC 2014, March 2014, pp. 2582-2587.

[21] C. Felgemacher, S. V. Araújo, P. Zacharias, K. Nesemann, and A. Gruber, "Cosmic radiation ruggedness of si and sic power semiconductors," in 2016 28th International Symposium on Power Semiconductor Devices and ICs (ISPSD), June 2016, pp. 51-54.

[22] S. Bozhko, T. Yang, J. Le Peuvedic, P. Arumugam, M. Degano, A. La Rocca, Z. Xu, M. Rashed, W. Fernando, C. I. Hill, C. Eastwick, S. Pickering, C. Gerada, and P. Wheeler, "Development of aircraft electric starter-generator system based on active rectification technology," IEEE Transactions on Transportation Electrification, vol. 4, no. 4, pp. 985996, Dec 2018.

[23] J. Harikumaran, G. Buticchi, M. Galea, A. Costabeber, and P. Wheeler, "Reliability analysis of aircraft starter generator drive converter," in 2019 IEEE 15th Brazilian Power Electronics Conference and 5th IEEE Southern Power Electronics Conference (COBEP/SPEC), 2019, pp. 1-6.

[24] N. Oceanic and A. Administration, U.S. Standard Atmosphere, 1976, National Oceanic and Atmospheric Administration, 1976.

[25] A. Wintrich, U. Nicolai, W. Tursky, and T. Reimann, Application Manual Power Semiconductors, SEMIKRON International GmbH, 2015. 